\title{
A Semigroup Formulation for Electromagnetic Waves in Dispersive Dielectric Media
}

\author{
H.T. Banks \\ Center for Research in Scientific Computation \\ NC State University \\ Raleigh, NC. 27695-8205
}

M.W. Buksas

Los Alamos National Laboratory

T-CNLS MS B258

Los Alamos, NM. 87545

November 8, 1999 


\begin{abstract}
We consider Maxwell systems with instantaneous conductivity and nonlocal in time (hysteretic) polarization laws which are characteristic of dispersive dielectric media. We formulate such systems in an operator theoretic framework and show that, under certain conditions on the dielectric response function, the resulting systems generate $C_{0}$ semigroups. It is shown that multiple Debye polarization models are included in those for which a semigroup formulation is possible.
\end{abstract}




\section{Introduction}

In a forthcoming monograph [BBL00] we have developed a theoretical and computational framework for electromagnetic interrogation of dispersive dielectric media. In that work we show that one can take a time domain variational or weak formulation of Maxwell's equations in dispersive materials and, in the context of inverse problems, use partially reflected polarized microwave pulses to determine both dielectric material properties and geometry of bodies (specifically for plane waves inpinging on slab geometries in paradyms which approximate far field interrogation). This is done in configurations involving either supraconductive reflecting back boundaries or acoustically generated virtual reflectors.

The propagation and reflection of electromagnetic waves in dispersive dielectric media is, of itself, an interesting topic of investigation. As we point out in the next section (and demonstrate computationally in [BBL00]), the underlying dynamical systems are not typical of either standard parabolic or standard hyperbolic (even with the usual dissipation) systems and are hence of mathematical interest. In this short note, we consider the Maxwell system for rather general dispersive dielectric media and show that such systems, under appropriate conditions on the polarization law, generate $C_{0}$ semigroup solutions. These results are presented in the context of the 1-dimensional interrogating systems developed in detail in [BBL00] and we invite interested readers to consult that reference for more detailed discussions and development of the underlying model employed here. 


\section{Modeling of Dispersiveness in Dielectric Media}

We begin with time domain Maxwell's equations in second order form (e.g., see [BBL00]) for the electric field $E=E(t, z)$ of 1-dimensional polarized waves

$$
\ddot{E}+\frac{1}{\epsilon_{0}} \ddot{P}+\frac{1}{\epsilon_{0}} \dot{J}_{c}-c^{2} E^{\prime \prime}=-\frac{1}{\epsilon_{0}} \dot{J}_{s}
$$

where $c=\frac{1}{\sqrt{\epsilon_{0} \mu_{0}}}$ is the speed of light in vacuum, $J_{c}$ is the conduction current density, $J_{s}$ is a source current density and $P$ is the electric polarization of the dielectric medium. We assume very general constitutive material laws for the polarization and conductivity given by

$$
\begin{aligned}
& P(t, z)=\left(g_{p} * E\right)(t, z)=\int_{0}^{t} g_{p}(t-s, z) E(s, z) d z \\
& J_{c}(t, z)=\left(g_{c} * E\right)(t, z)=\int_{0}^{t} g_{c}(t-s, z) E(s, z) d z
\end{aligned}
$$

where we have tacitly assumed that $E(t, z)=0$ for $t<0$ and that both $g_{p}(\xi, z)$ and $g_{c}(\xi, z)$ vanish for $\xi<0$. With these assumptions, the integrals in equations $(2.2)$, (2.3) are equivalent to integration over all of $(-\infty, \infty)$ and thus are indeed convolutions. The displacement susceptibility kernel $g_{p}$ (also referred to as the dielectric response function(DRF)) and the conductivity susceptibility kernel $g_{c}$ introduce nonlocality in time in the polarization and conductivity relationships [APM89], [Jac75] which is equivalent to frequency dependence of the dielctric permittivity $\epsilon$ and conductivity $\sigma$ when using a frequency domain approach. We assume that either $P$ or $J_{c}$ or both may contain instantaneous (local in time) components by introduction of $\delta$ distributions in the kernels $g_{p}$ and/or $g_{c}$, respectively.

A medium is dispersive if the phase velocity of plane waves propagating through it depends on the frequency of the waves [Jac75, Chap.7], [Ch89, Chap.8]. To determine the dispersive nature of a medium described by equations (2.1)-(2.3) we seek planewave solutions of the homogeneous analogue of (2.1) of the form $E(t, z)=E_{0} e^{-i(\omega t \pm \kappa z)}$ 
which travel in the $z$ direction and have wavelength $\lambda=2 \pi / \kappa$. The phase velocity $v_{p}$ of these waves is the speed at which planes of constant phase move through the medium. In this case the argument $\omega t-\kappa z$ is constant and

$$
v_{p}=\frac{d z}{d t}=\omega / \kappa
$$

Seeking plane wave solutions of the form $E_{0} e^{-i(\omega t \pm \kappa z)}$ in (2.1) is equivalent to seeking solutions of the form $E_{0} e^{ \pm i \kappa z}$ in the frequency domain version of (2.1). Thus we use the Fourier transform in (2.1) and obtain

$$
-\omega^{2} \hat{E}-\frac{\omega^{2}}{\epsilon_{0}} \hat{P}-\frac{i \omega}{\epsilon_{0}} \hat{J}_{c}-c^{2} \hat{E}^{\prime \prime}=0
$$

where we have ignored the source term $J_{s}$ and where the overhat will represent the Fourier transform throughout. Since we see from (2.2) and (2.3) that $\hat{P}=\hat{g}_{p} \hat{E}$ and $\hat{J}_{c}=\hat{g}_{c} \hat{E}$, this can be written

$$
c^{2} \hat{E}^{\prime \prime}+\omega^{2}\left(1+\frac{i \hat{g}_{c}}{\omega \epsilon_{0}}+\frac{\hat{g}_{p}}{\epsilon_{0}}\right) \hat{E}=0
$$

We note that (2.6) is the generalized Helmholtz equation [Jac75, p. 271]

$$
\hat{E}^{\prime \prime}+\kappa^{2} \hat{E}=0
$$

with

$$
\kappa^{2}=\frac{\omega^{2}}{c^{2}}\left(1+\frac{i \hat{g}_{c}(\omega)}{\omega \epsilon_{0}}+\frac{\hat{g}_{p}(\omega)}{\epsilon_{0}}\right)
$$

which has solutions $\hat{E}(\omega, z)=E_{0} e^{ \pm i \kappa(\omega) z}$. It follows that the corresponding time domain solutions are our desired solutions of the form $E(t, z)=E_{0} e^{-i(\omega t \pm i \kappa z)}$ where the wavenumber $\kappa=\kappa(\omega)$ will in general depend on the frequency $\omega$. The equation (2.8) relating the frequency $\omega$ and the wavenumber $\kappa$ of propagating waves is known as the dispersion equation for the medium. In the case of vacuum or free space where $\hat{g}_{p}=\hat{g}_{c}=0$ so that $\kappa=\omega / c$, we obtain the corresponding phase velocity $v_{p}=c=$ the 
speed of light as expected. More generally the phase velocity in a dielectric medium with conductivity and polarization is given by

$$
v_{p}=c / \sqrt{1+i \hat{g}_{c} / \epsilon_{0} \omega+\hat{g}_{p} / \epsilon_{0}}
$$

In light of (2.9) and the definition of a dispersive medium, we see that if either $\hat{g}_{c} / \omega$ or $\hat{g}_{p}$ depend on $\omega$, we will have dispersiveness. Several special cases are worthy of note.

For instantaneous conductivity, that is, $g_{c}(t, z)=\sigma \delta(t)$ so that (2.3) reduces to Ohm's Law $J_{c}=\sigma E$, we see that the term $i \hat{g}_{c} / \epsilon_{0} \omega$ becomes $i \sigma / \epsilon_{0} \omega$. Thus a medium with simple Ohm's Law conductivity will be dispersive (it is also dissipative in the usual sense since the conductivity term in (2.1) becomes $\frac{\sigma}{\epsilon_{0}} \dot{E}$ ). For instantaneous polarization (often assumed in standard treatments of the Maxwell theory) we find $g_{p}(t, z)=\epsilon_{0} \chi \delta(t)$, where $\chi$ is the dielectric susceptibility constant and hence $\hat{g}_{p} / \epsilon_{0}=\chi$ and the medium is not dispersive. One must turn to more complicated (and more realistic) models, such as those of Debye or Lorentz, to have a polarization based contribution to dispersiveness in a medium. For the usual Debye polarization model [Ell93, p.386] one has

$$
g_{p}(t)=e^{-t / \tau} \epsilon_{0}\left(\epsilon_{s}-\epsilon_{\infty}\right) / \tau, \quad t>0
$$

where $\tau$ is a relaxation parameter and $\epsilon_{s}, \epsilon_{\infty}$ are familiar dielectric constants. In this case one finds

$$
\hat{g}_{p}(\omega)=\epsilon_{0}\left(\epsilon_{s}-\epsilon_{\infty}\right)\left[\frac{1-i \omega \tau}{1+\tau^{2} \omega^{2}}\right]
$$

For the Lorentz model [RMC93, p.496] we have

$$
g_{p}(t)=\frac{\epsilon_{0} \omega_{p}^{2}}{\nu_{o}} e^{-t / 2 \tau} \sin \nu_{0} t, \quad t>0
$$


where $\nu_{0}=\sqrt{\omega_{0}^{2}-1 / 4 \tau^{2}}$. In the frequency domain this yields

$$
\begin{aligned}
\hat{g}_{p}(\omega) & =\epsilon_{0} \omega_{p}^{2}\left[\frac{4 \tau^{2}}{(1+i 2 \tau \omega)^{2}+4 \tau^{2} \nu_{0}^{2}}\right] \\
& =\epsilon_{0} \omega_{p}^{2} \nu_{0}\left[\frac{\left(\omega_{0}^{2}-\omega^{2}\right)-i \omega / \tau}{\left(\omega_{0}^{2}-\omega^{2}\right)^{2}+\omega^{2} / \tau^{2}}\right]
\end{aligned}
$$

and again we have a polarization based dispersive medium. Higher order (the Debye and Lorentz models correspond to first and second order, respectively, differential equation models for the polarization $P$ - see [BBL00] and the references therein) models, as well as combinations of such models also lead to dispersion in a medium.

Thus, in summary we see that instantaneous conductivity but not instantaneous polarization yields dispersiveness in a medium. For a polarization contribution to dispersiveness one must include first or higher order polarization models (instantaneous polarization can be correctly viewed as zero order polarization dynamics). For our semigroup presentation in the next section we shall therefore consider the model (2.1) with instantaneous conductivity and a general (higher order) polarization model given by (2.2) with $g_{p}=g$ where the DRF $g$ is assumed smooth in time (i.e., without loss of generality we can assume no instantaneous component for $g$ ). Such distributed parameter systems are of interest since they are neither simple hyperbolic nor parabolic in nature.

For simple Ohm's Law conductivity and instantaneous polarization (or no polarization), the system (2.1) becomes a well understood dissipative or damped hyperbolic system for which a semigroup formulation can readily be found in the research literature on distributed parameter systems. However, for (2.1) with polarization based dispersiveness, we obtain a system with behavior of solutions that are neither standard hyperbolic (finite speed of wave propagation along characteristics) nor standard parabolic (infinite speed of propagation of disturbances). Indeed for (2.1) with either Debye or Lorentz polarization, rather fascinating solutions can be observed. These in- 
volve the formulation of so-called Brillouin and Sommerfeld precursors where a pulsed excitation (containing waves with a range of frequencies) evolves into waves propagated with different velocities which coalesce into wave "packets" (see Chapter 4 of [BBL00] and [APM89] and the references therein for discussions of these phenomena).

It is of both mathematical and practical interest to know whether these interesting systems can be described in a semigroup context. The potential advantages afforded by a semigroup formulation are widespread since there is a tremendous literature for control, estimation and identification, and stabilization of systems in a semigroup setting. Results for both stochastic and deterministic control methodologies (in both time domain and frequency domain) including open loop and feedback formulations are abundant [CZ95], [BDDM92], [BDDM93], [CP78], [vK93].

In the next section we present a semigroup formulation of the system (2.1) with simple Ohm's Law conductivity along with general polarization based dispersiveness generated by polarization laws of the form (2.2). To be more precise, we take (2.1) for $t>0$ and $z \in(0,1)$ with $J_{c}(t, z)=\sigma(z) E(t, z)$ where $\sigma(z)$ vanishes outside $\Omega \subset(0,1]$. The closed region $\Omega$ is some dielectric material region (e.g., a slab or several slab-like regions) containing instantaneous conductivity as well as non trivial polarization of the form (2.2) with $g_{p}(t, z)=g(t, z)$ vanishing outside $z \in \Omega$. Using this form of conductivity and polarization in (2.1), we obtain the system

$$
\begin{array}{r}
\ddot{E}(t, z)+\frac{1}{\epsilon_{0}}(\sigma(z)+g(0, z)) \dot{E}(t, z)+\frac{1}{\epsilon_{0}} \dot{g}(0, z) E(t, z) \\
+\int_{0}^{t} \frac{1}{\epsilon_{0}} \ddot{g}(t-s, z) E(s, z) d s-c^{2} E^{\prime \prime}(t, z)=-\frac{1}{\epsilon_{0}} \dot{J}_{s}(t, z) .
\end{array}
$$

With this system we take boundary conditions (see [BBL00] for details) that represent a total absorbing boundary at $z=0$ and a supraconductive boundary at $z=1$. This can be expressed by

$$
\dot{E}(t, 0)-c E^{\prime}(t, 0)=0
$$




$$
E(t, 1)=0 .
$$

With the definitions

$$
\begin{aligned}
\alpha(t, z) & =\frac{1}{\epsilon_{0}} \ddot{g}(t, z), \quad \beta(z)=\frac{1}{\epsilon_{0}} \dot{g}(0, z) \\
\gamma(z) & =\frac{1}{\epsilon_{0}}(\sigma(z)+g(0, z)), \quad \mathcal{J}(t, z)=-\frac{1}{\epsilon_{0}} \dot{J}_{s}(t, z),
\end{aligned}
$$

we can write equation (2.12) as

$$
\ddot{E}+\gamma \dot{E}+\beta E+\alpha * E-c^{2} E^{\prime \prime}=\mathcal{J}
$$

where $\alpha * E$ is the usual convolution

$$
\alpha * E(t, z)=\int_{0}^{t} \alpha(t-s, z) E(s, z) d s .
$$

One can use the boundary conditions (2.13) - (2.14) to write (2.15) in weak or variational form so as to seek solutions $t \rightarrow E(t)$ in $V \equiv H_{R}^{1}(0,1)=\left\{\phi \in H^{1}(0,1)\right.$ : $\phi(1)=0\}$ in a Gelfand triple setting $V \hookrightarrow H \hookrightarrow V^{*}$ with pivot space $H=L^{2}(0,1)$. Under modest regularity assumptions on $\alpha, \beta, \gamma$ and $\mathcal{J}$, one can establish existence, uniqueness and continuous dependence (on initial conditions and input) of solutions. Details are given in Chapter 3 of [BBL00]. 


\section{A Semigroup Formulation}

We turn in this section to a semigroup formulation for the dispersive system (2.12) - (2.14) or equivalently, (2.13) - (2.15), with instantaneous conductivity and general (non instantaneous) polarization.

For our development we assume that $\gamma, \beta \in L^{\infty}(0,1)$ while $\alpha \in L^{\infty}((0, T) \times$ $(0,1))$ and $\alpha, \beta, \gamma$ vanish outside $\Omega$. We moreover assume that $\alpha$ can be written as $\alpha(t, z)=\alpha_{1}(t) \alpha_{2}(z)$ where $0<\alpha_{L} \leq \alpha_{2}(z) \leq \alpha_{U}$ on $\Omega \subset(0,1]$ for positive constants $\alpha_{L}, \alpha_{U}$, with $\alpha_{2}$ vanishing outside $\Omega$. We assume that $t \rightarrow \alpha_{1}(t)$ is positive, monotone nonincreasing, and in $H^{1}(0, T)$ so that $\dot{\alpha}_{1}(t) \leq 0$. This monotonicity assumption is typical of the usual assumptions in displacement susceptibility kernels (e.g., see [Blo81, p.102] or [Hop77]). We shall return to discuss this monotonicity requirement further after our semigroup arguments of this section.

We consider the term (2.16) given by

$$
\int_{0}^{t} \alpha(t-s) E(s) d s=\int_{-\infty}^{t} \alpha(t-s) E(s) d s
$$

from (2.15) and note that it can be equivalently written

$$
\begin{aligned}
& \int_{-\infty}^{t} \alpha(t-s) E(s) d s=\int_{-\infty}^{0} \alpha(-\xi) E(t+\xi) d \xi \\
& \quad \approx \int_{-r}^{0} \alpha(-\xi) E(t+\xi) d \xi=\int_{-r}^{0} G(\xi) E(t+\xi) d \xi
\end{aligned}
$$

where $G(\xi) \equiv \alpha(-\xi)$. We denote $G_{1}(\xi)=\alpha_{1}(-\xi)$ so that $G(\xi)=G_{1}(\xi) \alpha_{2}$.

The approximation is valid for $r$ sufficiently large $(r=\infty$ is permitted) so that $\alpha(t) \approx 0$ for $t>r$. We observe at this point that $\dot{G}_{1}(\xi) \geq 0$ with $G_{1}(\xi)>0$ on $(-r, 0]$.

As introduced in the previous section, we take $V=H_{R}^{1}(0,1), H=L^{2}(0,1)$ with $V \hookrightarrow H \hookrightarrow V^{*}$. We shall have use of $\tilde{H}=L_{\alpha_{2}}^{2}(\Omega)$, the space $L^{2}(\Omega)$ with weighting function $\alpha_{2}$, which is readily seen to be equivalent to $L^{2}(\Omega)$ due to the upper and lower bounds on $\alpha_{2} \in L^{\infty}(\Omega)$. We shall denote the restriction of functions $\phi$ in $L^{2}(0,1)$ to $\Omega$ again by $\phi$ and write $\phi \in L^{2}(\Omega)$ or $\phi \in L_{\alpha_{2}}^{2}(\Omega)$ whenever no confusion will result. 
Using the above definitions and approximating, we may write (2.15) as

$$
\ddot{E}(t)+\gamma \dot{E}(t)+\beta E(t)+\int_{-r}^{0} G(\xi) E(t+\xi) d \xi-c^{2} E^{\prime \prime}(t)=\mathcal{J}(t)
$$

Using an approach given in [BFW88], [BFW89], [FI90] and [BMZ96] for viscoelastic systems, we define an auxiliary variable $w(t)$ in $W \equiv L_{G_{1}}^{2}(-r, 0 ; \tilde{H})$ by $w(t)(\theta)=$ $E(t)-E(t+\theta),-r \leq \theta \leq 0$. Since $G(\theta, z)>0$ for $\theta \in(-r, 0], z \in \Omega$ we may take as an inner product for $W$ the weighted $L^{2}$ inner product

$$
\langle\eta, w\rangle_{W} \equiv \int_{-r}^{0} G_{1}(\theta)\langle\eta(\theta), w(\theta)\rangle_{\tilde{H}} d \theta=\int_{-r}^{0} G_{1}(\theta) \int_{\Omega} \alpha_{2}(z) \eta(\theta, z) w(\theta, z) d z
$$

under which $W$ is a Hilbert Space. We note that by our notational convention explained above, we have $w(t) \in W$ for any $E(t, z)$ with $E(\cdot, \cdot) \in L_{G_{1}}^{2}(-r, 0 ; H)$. Using a standard shift notation, we may write $w(t)=E(t)-E(t+\theta)=E(t)-E^{t}(\theta)$ where $E^{t}(\theta) \equiv E(t+\theta)$ for $-r \leq \theta \leq 0$. Adding and subtracting appropriate terms in (3.1), we find

$\ddot{E}(t)+\gamma \dot{E}(t)+\beta E(t)+\int_{-r}^{0} G(\xi) E(t) d \xi-\int_{-r}^{0} G(\xi)\left[E(t)-E^{t}(\xi)\right] d \xi-c^{2} E^{\prime \prime}(t)=\mathcal{J}(t)$ or, equivalently

$$
\ddot{E}(t)+\gamma \dot{E}+\left(\beta+G_{11}\right) E(t)-\int_{-r}^{0} G(\xi) w(t)(\xi) d \xi-c^{2} E^{\prime \prime}(t)=\mathcal{J}(t)
$$

where $G_{11}(z) \equiv \int_{-r}^{0} G(\xi) d \xi=\alpha_{2}(z) \int_{-r}^{0} G_{1}(\xi) d \xi$ and $w(t)(\xi)=E(t)-E^{t}(\xi)$. We observe that $G_{11}$, like $\beta$, is in $L^{2}(\Omega)$ and $L^{2}(0,1)$.

For our semigroup formulation, we consider (3.2) in the state space $Z=V \times H \times$ $W=H_{R}^{1}(0,1) \times L^{2}(0,1) \times L_{G_{1}}^{2}(-r, 0, \tilde{H})$ with states $(\phi, \psi, \eta)=(E(t), \dot{E}(t), w(t))=$ $\left(E(t), \dot{E}(t), E(t)-E^{t}(\cdot)\right)$. To define an infinitesimal generator, we begin by defining a fundamental set of component operators. Let $\tilde{A} \in \mathcal{L}\left(V, V^{*}\right)$ be defined by

$$
\tilde{A} \phi \equiv c^{2} \phi^{\prime \prime}-\left(\beta+G_{11}\right) \phi+c^{2} \phi^{\prime}(0) \delta_{0}
$$


where $\delta_{0}$ is the Dirac operator $\delta_{0} \psi=\psi(0)$. Then we find

$$
\langle-\tilde{A} \phi, \psi\rangle_{V^{*}, V}=\left\langle c^{2} \phi^{\prime}, \psi^{\prime}\right\rangle_{H}+\left\langle\left(\beta+G_{11}\right) \phi, \psi\right\rangle_{H}
$$

so that it is readily seen that $\tilde{\sigma}_{1}: V \times V \mapsto \mathbb{C}$ defined by

$$
\tilde{\sigma}_{1}(\phi, \psi)=\langle-\tilde{A} \phi, \psi\rangle_{V^{*}, V}
$$

is symmetric, $V$ continuous and $V$ coercive (i.e., $\tilde{\sigma}_{1}(\phi, \phi) \geq c_{1}|\phi|_{V}^{2}-\lambda_{0}|\phi|_{H}^{2}$ for constants $\lambda_{0}$ and $\left.c_{1}>0\right)$.

We also define operators $B \in \mathcal{L}\left(V, V^{*}\right)$ and $\tilde{K} \in \mathcal{L}(W, H)$ by

$$
B \phi=-\gamma \phi-c \phi(0) \delta_{0}
$$

so that

$$
\langle-B \phi, \psi\rangle_{V^{*}, V}=\langle\gamma \phi, \psi\rangle_{H}+c \phi(0) \psi(0)
$$

and, for $\eta \in W=L_{G_{1}}^{2}(-r, 0 ; \tilde{H})$,

$$
(\tilde{K} \eta)(z)= \begin{cases}0 & z \in[0,1] \backslash \Omega \\ \int_{-r}^{0} G(\xi) \eta(\xi) d \xi & z \in \Omega .\end{cases}
$$

Since $G(\xi, z)=0$ for $z \in[0,1] \backslash \Omega$, we abuse notation and write this as

$$
\tilde{K} \eta=\int_{-r}^{0} G(\xi) \eta(\xi) d \xi
$$

even though, strictly speaking, $\eta(\xi, z)$ is only defined for $z \in \Omega$.

With these definitions and notations, equation (3.2) can then be written as

$$
\begin{aligned}
\langle\ddot{E}, \phi\rangle_{V^{*}, V} & +\langle-\tilde{A} E, \phi\rangle_{V^{*}, V}+\langle-B \dot{E}, \phi\rangle_{V^{*}, V} \\
+ & \left\langle-\tilde{K}\left(E-E^{t}\right), \phi\right\rangle_{V^{*}, V}=\langle\mathcal{J}, \phi\rangle_{V^{*}, V}
\end{aligned}
$$

or

$$
\ddot{E}(t)=\tilde{A} E(t)+B \dot{E}(t)+\tilde{K}\left(E(t)-E^{t}\right)+\mathcal{J}(t) \text { in } V^{*}
$$


We rewrite equation (3.9) as a first order system in the state $\zeta(t)=(E(t), \dot{E}(t), w(t))$ where $w(t)=E(t)-E^{t}$. To aid in this we introduce another operator

$$
D: \operatorname{dom} D \subset W \mapsto W
$$

defined on $\operatorname{dom} D=\left\{\eta \in H^{1}(-r, 0 ; \tilde{H}) \mid \eta(0)=0\right\}$ by

$$
D \eta(\theta)=\frac{\partial}{\partial \theta} \eta(\theta)
$$

We then observe that $w(t)=E(t)-E^{t}$ satisfies

$$
\begin{aligned}
\dot{w}(t)(\theta) & =\dot{E}(t)-\dot{E}(t+\theta)=\dot{E}(t)-D E^{t}(\theta) \\
& =\dot{E}(t)+D\left(E(t)-E^{t}(\theta)\right)=\dot{E}(t)+D w(t)(\theta)
\end{aligned}
$$

Thus we may formally rewrite (3.9) as a first order system and adjoin to it the equation

$$
\dot{w}(t)=D w(t)+\dot{E}(t)
$$

We then obtain the first order system for $\zeta(t)$ given by

$$
\dot{\zeta}(t)=\mathcal{A} \zeta(t)+\mathcal{F}(t)
$$

where $\mathcal{A}$ given by

$$
\mathcal{A}=\left(\begin{array}{ccc}
0 & I & 0 \\
\tilde{A} & B & \tilde{K} \\
0 & I & D
\end{array}\right)
$$

is defined on

$$
\operatorname{dom} \mathcal{A}=\{(\phi, \psi, \eta) \in Z \mid \psi \in V, \eta \in \operatorname{dom} D, \tilde{A} \phi+B \psi \in H\}
$$

That is, $\mathcal{A} \Phi=(\phi, \tilde{A} \phi+B \psi+\tilde{K} \eta, \psi+D \eta)$ for $\Phi=(\phi, \psi, \eta)$ in dom $\mathcal{A}$. The forcing function $\mathcal{F}$ in $(3.11)$ is given by $\mathcal{F}=\operatorname{col}(0, \mathcal{J}, 0)$. To argue that $\mathcal{A}$ is the infinitesimal 
generator of a $C_{0}$-semigroup, we actually consider the system (3.11) on an equivlaent space $Z_{1}=V_{1} \times H \times W$ where $V_{1}$ is the space $V$ with equivalent inner product $\left\langle\phi_{1}, \phi_{2}\right\rangle_{V_{1}}=\tilde{\sigma}_{1}\left(\phi_{1}, \phi_{2}\right)$ where $\tilde{\sigma}_{1}$ is the sesquilinear form given in (3.5). Recall that $\tilde{\sigma}_{1}$ is symmetric, $V$ continuous and $V$ coercive so that it is topologically equivalent to the $V$ inner product.

We are now ready to prove the following generation theorem.

Theorem Suppose that $\gamma, \beta \in L^{\infty}(0,1), \alpha \in L^{\infty}((0,1) \times(0,1))$ with $\alpha, \beta, \gamma$ vanishing outside $\Omega$. We further assume that $\alpha$ can be written $\alpha(t, z)=\alpha_{1}(t) \alpha_{2}(z)$ where $\alpha_{1} \in H^{1}(0, T)$ with $\alpha_{1}(t)>0, \dot{\alpha}_{1}(t) \leq 0$, and $0<\alpha_{L} \leq \alpha_{2}(z) \leq \alpha_{U}$ for positive constants $\alpha_{L}, \alpha_{U}$. Then the operator $\mathcal{A}$ defined by (3.12), (3.13) is the infinitesimal generator of a $C_{0}$-semigroup on $Z_{1}$ and hence on the equivalent space $Z$.

To prove this theorem, we use the Lumer-Phillips theorem ([Paz83, p.14]). Since $Z_{1}$ is a Hilbert space, it suffices to argue that for some $\lambda_{0}, \mathcal{A}-\lambda_{0} I$ is dissipative in $Z_{1}$ and $\mathcal{R}(\lambda I-\mathcal{A})=Z_{1}$ for some $\lambda>0$, where $\mathcal{R}(\lambda I-\mathcal{A})$ is the range of $\lambda I-\mathcal{A}$. We first argue dissipativeness.

Let $\Phi=(\phi, \psi, \eta) \in \operatorname{dom} \mathcal{A}$. Then

$$
\begin{aligned}
\langle A \Phi, \Phi\rangle_{Z_{1}} & =\langle\psi, \phi\rangle_{V_{1}}+\langle\tilde{A} \phi+B \psi+\tilde{K} \eta, \psi\rangle_{H}+\langle\psi+D \eta, \eta\rangle_{W} \\
& =\langle\psi, \phi\rangle_{V_{1}}+\langle\tilde{A} \phi+B \psi, \psi\rangle_{V^{*}, V}+\langle\tilde{K} \eta, \psi\rangle_{H}+\langle\psi+D \eta, \eta\rangle_{W} \\
& =\tilde{\sigma}_{1}(\psi, \phi)-\tilde{\sigma}_{1}(\phi, \psi)+\langle B \psi, \psi\rangle_{H}+\langle\tilde{K} \eta, \psi\rangle_{H}+\langle\psi+D \eta, \eta\rangle_{W} \\
& =-\langle\gamma \psi, \psi\rangle_{H}-c|\psi(0)|^{2}+\langle\tilde{K} \eta, \psi\rangle_{H}+\langle\psi+D \eta, \eta\rangle_{W} \\
& \leq|\gamma|_{\infty}|\psi|_{H}^{2}+\left|\langle\tilde{K} \eta, \psi\rangle_{H}\right|+\left|\langle\psi+D \eta, \eta\rangle_{W}\right|
\end{aligned}
$$

We consider estimates for the last two terms in (3.14) separately. From (3.8) we have

$$
\left|\langle\tilde{K} \eta, \psi\rangle_{H}\right|=\left|\int_{-r}^{0} G_{1}(\theta)\langle\eta(\theta), \psi\rangle_{\tilde{H}} d \theta\right|
$$




$$
\begin{aligned}
& \leq \int_{-r}^{0} G_{1}(\theta)|\eta(\theta)|_{\tilde{H}}|\psi|_{\tilde{H}} d \theta \\
& \leq \frac{1}{2} \int_{-r}^{0} G_{1}(\theta)\left\{|\eta(\theta)|_{\tilde{H}}^{2}+|\psi|_{\tilde{H}}^{2}\right\} d \theta \\
& \leq k_{1}|\eta|_{W}^{2}+k_{2}|\psi|_{H}^{2} .
\end{aligned}
$$

Moreover,

$$
\begin{aligned}
\left|\langle\psi, \eta\rangle_{W}\right| & \leq \int_{-r}^{0} G_{1}(\theta)\left|\langle\psi, \eta(\theta)\rangle_{\tilde{H}}\right| d \theta \\
& \leq \int_{-r}^{0} G_{1}(\theta)\left\{\frac{1}{2}|\psi|_{\tilde{H}}^{2}+\frac{1}{2}|\eta(\theta)|_{\tilde{H}}^{2}\right\} d \theta \\
& \leq k_{3}|\psi|_{H}^{2}+k_{4}|\eta|_{W}^{2} .
\end{aligned}
$$

Finally, since $G_{1}(\theta) \geq 0, \dot{G}_{1} \geq 0$, and $\eta \in \operatorname{dom} D$ requires $\eta(0)=0$, we may argue

$$
\begin{aligned}
\langle D \eta, \eta\rangle_{W} & =\int_{-r}^{0} G_{1}(\theta)\langle D \eta(\theta), \eta(\theta)\rangle_{\tilde{H}} d \theta \\
& =\int_{-r}^{0} G_{1}(\theta) \frac{d}{d \theta} \frac{1}{2}|\eta(\theta)|_{\tilde{H}}^{2} d \theta \\
& =\int_{-r}^{0} \frac{d}{d \theta}\left(G_{1}(\theta) \frac{1}{2}|\eta(\theta)|_{\tilde{H}}^{2}\right) d \theta-\int_{-r}^{0} \frac{1}{2}\left(\dot{G}_{1}(\theta)|\eta(\theta)|_{\tilde{H}}^{2}\right) d \theta \\
& =G_{1}(0) \frac{1}{2}|\eta(0)|_{\tilde{H}}^{2}-G_{1}(-r) \frac{1}{2}|\eta(-r)|_{\tilde{H}}^{2}-\frac{1}{2} \int_{-r}^{0} \dot{G}_{1}(\theta)|\eta(\theta)|_{\tilde{H}}^{2} d \theta \\
& \leq 0 .
\end{aligned}
$$

Combining these estimates with (3.14), we obtain for $\Phi \in \operatorname{dom} \mathcal{A}$

$$
\begin{aligned}
\langle\mathcal{A} \Phi, \Phi\rangle_{Z_{1}} & \leq|\gamma|_{\infty}|\psi|_{H}^{2}+\left(k_{1}+k_{4}\right)|\eta|_{W}^{2}+\left(k_{2}+k_{3}\right)|\psi|_{H}^{2} \\
& \leq \lambda_{0}|\Phi|_{Z_{1}}^{2}
\end{aligned}
$$

which yields the desired dissipativeness in $Z_{1}$.

To establish the range statement, we must argue there exists some $\lambda>0$ such that for any given $\Psi=(\mu, \nu, \xi)$ in $Z$, there exists $\Phi$ in dom $\mathcal{A}$ satisfying

$$
(\lambda I-\mathcal{A}) \Phi=\Psi
$$


In view of the definition of $\mathcal{A}$, the equation (3.15) is equivalent to the system

$$
\begin{aligned}
\lambda \phi-\psi & =\mu \\
-\tilde{A} \phi+(\lambda-B) \psi-\tilde{K} \eta & =\nu \\
-\psi+(\lambda-D) \eta & =\xi
\end{aligned}
$$

for $(\phi, \psi, \eta) \in \operatorname{dom} \mathcal{A},(\mu, \nu, \xi) \in Z=V \times H \times W$. The first equation is the same as $\psi=$ $\lambda \phi-\mu$ while the third can be written as $\eta=(\lambda-D)^{-1}(\xi+\psi)=(\lambda-D)^{-1}(\xi+\lambda \phi-\mu)$. These two equations can be substituted in the second to obtain an equation for $\phi$. If this equation can be solved for $\phi \in V$, then the first and third can then be solved for $\psi$ and $\eta$, respectively. The equation for $\phi$ that must be solved is given by

$$
-\tilde{A} \phi+(\lambda-B)(\lambda \phi-\mu)-\tilde{K}(\lambda-D)^{-1}(\xi+\lambda \phi-\mu)=\nu
$$

or

$$
\left[\lambda^{2}-\lambda B-\tilde{A}-\tilde{K}(\lambda-D)^{-1} \lambda\right] \phi=(\lambda-B) \mu+\nu+\tilde{K}(\lambda-D)^{-1}(\xi-\mu) .
$$

If we can invert (3.17) for $\phi \in V$, then $\psi=\lambda \phi-\mu$ is in $V, \eta=(\lambda-D)^{-1}[\xi+\lambda \phi-\mu]$ is in $\operatorname{dom} D \subset W$ and

$$
\begin{aligned}
\tilde{A} \phi+B \psi & =\lambda^{2} \phi-\lambda \mu-\nu-\tilde{K}(\lambda-D)^{-1}(\xi+\lambda \phi-\mu) \\
& =\lambda \psi-\nu-\tilde{K} \eta
\end{aligned}
$$

is in $H$ so that $(\phi, \psi, \eta)$ is in $\operatorname{dom} \mathcal{A}$ and solves (3.16).

Thus the range statement reduces to solving (3.17) for $\phi \in V$. This in turn reduces to invertability of the operator $\lambda^{2}-\lambda B-\tilde{A}-\tilde{K}(\lambda-D)^{-1} \lambda$.

We first observe that $(\lambda-D)^{-1}=\left(1-e^{\lambda \cdot}\right) / \lambda$ since $(\lambda-D)\left(1-e^{\lambda \theta}\right)=\lambda$ while $\eta(\theta)=\frac{1-e^{\lambda \theta}}{\lambda}[\xi+\lambda \phi-\mu]$ satisfies $\eta(0)=0$ and hence is in $\operatorname{dom} D$.

Thus, for $\phi \in H, \quad \tilde{K}(\lambda-D)^{-1} \lambda$ satisfies

$$
\begin{aligned}
\left\langle\tilde{K}\left(1-e^{\lambda \theta}\right) \phi, \phi\right\rangle_{H} & =\int_{-r}^{0} G_{1}(\theta)\left(1-e^{\lambda \theta}\right)\langle\phi, \phi\rangle_{\tilde{H}} d \theta \\
& \leq k_{5}|\phi|_{H}^{2}
\end{aligned}
$$


and

$$
\begin{aligned}
\left\langle\left(\lambda^{2}-\lambda B\right) \phi, \phi\right\rangle_{V^{*}, V} & =\left\langle\left(\lambda^{2}+\lambda \gamma\right) \phi+\lambda c \phi(0) \delta_{0}, \phi\right\rangle_{V^{*}, V} \\
& =\left\langle\left(\lambda^{2}+\lambda \gamma\right) \phi, \phi\right\rangle_{H}+\lambda c|\phi(0)|^{2} \\
& \geq k_{6}|\phi|_{H}^{2} \quad \text { for } \lambda \text { sufficiently large. }
\end{aligned}
$$

Hence for $\lambda$ sufficiently large we have

$$
\begin{aligned}
\left\langle\left(\lambda^{2}-\lambda B\right.\right. & \left.\left.-\tilde{A}-\tilde{K}(\lambda-D)^{-1} \lambda\right) \phi, \phi\right\rangle_{V^{*}, V} \\
& =\left\langle\left(\lambda^{2}-\lambda B\right) \phi, \phi\right\rangle_{V^{*}, V}+\tilde{\sigma}_{1}(\phi, \phi)-\left\langle\tilde{K}(\lambda-D)^{-1} \lambda \phi, \phi\right\rangle_{H} \\
& \geq k_{6}|\phi|_{H}^{2}+c_{1}|\phi|_{V}^{2}-\lambda_{0}|\phi|_{H}^{2}-k_{5}|\phi|_{H}^{2} \\
& =c_{1}|\phi|_{V}^{2}+\left(k_{6}-\lambda_{0}-k_{5}\right)|\phi|_{H}^{2}
\end{aligned}
$$

Thus if we define the sesquilinear form

$$
\sigma_{\lambda}(\phi, \psi) \equiv\left\langle\left(\lambda^{2}-\lambda B-\tilde{A}-\tilde{K}(\lambda-D)^{-1} \lambda\right) \phi, \psi\right\rangle_{V^{*}, V},
$$

we see that for $\lambda$ sufficiently large, $\sigma_{\lambda}$ is $V$ coercive and hence, by the Lax-Milgram lemma [Wlo87], it is invertible. It follows immediately that (3.17) is invertible for $\phi \in V$. This completes the arguments to prove the Theorem.

Let $S(t)$ denote the semigroup generated by $\mathcal{A}$ so that solutions to (3.11) are given by

$$
\zeta(t)=S(t) \zeta_{0}+\int_{0}^{t} S(t-s) \mathcal{F}(s) d s .
$$

Solutions are clearly continuously dependent on initial data $\zeta_{0}$ and the nonhomogeneous perturbation $\mathcal{F}$. The first component of $\zeta(t)$ is a generalized solution $E(t)$ of (3.1). One can now argue that the solution agrees with the unique weak solution obtained in Chapter 3 of [BBL00], by using the arguments in Chapter 4.4 of [BSW96]. Briefly, one argues equivalence for sufficiently regular initial data and nonhomogeneous perturbation. Then density along with continuous dependence is used to extend the equivalence to more general data (see [BSW96] for details). 


\section{Concluding Remarks}

In the previous section we presented a semigroup generation theorem under general conditions on the coefficients $\alpha, \beta, \gamma$ of (2.15). The only possibly restrictive condition involved $\alpha(t, z)=\frac{1}{\epsilon_{0}} \ddot{g}(t, z)=\alpha_{1}(t) \alpha_{2}(z)$ where it is required that $\alpha_{1}(t)>0, \dot{\alpha_{1}}(t) \leq 0$. We consider more closely the condition for some common polarization laws.

For Debye polarization in a region $\Omega$, we have $\alpha_{1}(t)=\frac{1}{\epsilon_{0}} \ddot{g}_{p}(t)$ where $g_{p}$ is given in (2.10). That is,

$$
g_{p}(t)=\epsilon_{0} \frac{\left(\epsilon_{s}-\epsilon_{\infty}\right)}{\tau} e^{-t / \tau}
$$

so that

$$
\alpha_{1}(t)=\frac{1}{\epsilon_{0}} \ddot{g}_{p}(t)=\frac{\left(\epsilon_{s}-\epsilon_{\infty}\right)}{\tau^{3}} e^{-t / \tau}>0
$$

and

$$
\dot{\alpha_{1}}(t)=-\frac{\left(\epsilon_{s}-\epsilon_{\infty}\right)}{\tau^{4}} e^{-t / \tau}<0
$$

Thus Debye polarization satisfies the conditions of the generation theorem and the associated system generates a $C_{0}$ semigroup.

For Lorentz polarization, we have (recall (2.11))

$$
g_{p}(t)=\frac{\epsilon_{0} \omega_{p}^{2}}{\nu_{0}} e^{-t / 2 \tau} \sin \nu_{0} t
$$

and hence

$$
\alpha_{1}(t)=\frac{1}{\epsilon_{0}} \ddot{g}_{p}(t)=\frac{\omega_{p}^{2}}{\nu_{0}}\left[\left(\frac{1}{2 \tau^{2}}-\omega_{0}^{2}\right) \sin \nu_{0} t-\frac{\nu_{0}}{\tau} \cos \nu_{0} t\right]
$$

We therefore see that it is not possible to conclude that $\alpha_{1}(t)>0$ or $\dot{\alpha}_{1}(t) \leq 0$ so that our generation theorem does not guarantee a semigroup representation for systems with a Lorentz polarization law. In spite of this, we do believe that the Lorentz 
law does yield a system with a semigroup representation. We conjecture that the proof of the theorem we present can be modified to weaken the hypothesis on $\alpha$ so as to include Lorentz and other oscillatory (even order) polarization models. We are currently pursuing these ideas.

In closing we point out that the general class of dielectric response functions consisting of a linear combination of decreasing exponentials (essentially multiple Debye mechanisms) suggested for glasseous materials by Hopkinson [Hop77] (see the discussion in [Blo81, p.101-103]) are included under the theory presented in this note.

Acknowledgements: This research was supported in part by the Air Force Office of Scientific Research under grants AFOSR F49620-98-1-0180 and AFOSR F49620-951-0447 and the Department of Energy, under contract W-7405-ENG-36. The authors are grateful to Dr. Richard Albanese, U.S. Air Force Research Laboratory, Brooks AFB, San Antonio, TX, for his continued encouragement and numerous specific technical disscussions throughout the course of the research reported on here and in [BBL00]. 


\section{References}

[APM89] R. Albanese, J. Penn and R. Medina, Short-rise-time microwave pulse propagation through dispersive biological media, J. of Optical Society of America A, 6:1441-1446, 1989.

[BBL00] H.T. Banks, M.W. Buksas, and T. Lin, Electromagnetic Interrogation of Dielectric Materials, SIAM Frontiers in Applied Mathematics, SIAM, Philadelphia, 2000, to appear.

[BDDM92] A.Bensoussan, G. DaPrato, M.C. Delfour, and S.K. Mitter, Representation and Control of Infinite Dimensional Systems, Vol. I, Birkhäuser, Boston, 1992.

[BDDM93] A. Bensoussan, G. DaPrato, M.C. Delfour, and S.K. Mitter, Representation and Control of Infinite Dimensional Systems, Vol. II, Birkhäuser, Boston, 1993.

[BFW88] H.T. Banks, R.H. Fabiano and Y. Wang, Estimation of Boltzmann damping coefficients in beam models, In COMCON Conf.on Stabilization of Flexible Structures, pages 13-35, New York, 1988, Optimization Software, Inc.

[BFW89] H.T. Banks, R.H. Fabiano and Y. Wang, Inverse problem techniques for beams with tip body and time hysteresis damping, Mat. Aplic. e Comput., 8:101-118, 1989.

[Blo81] F. Bloom, Ill-Posed Problems for Integrodifferential Equations in Mechanics and Electromagnetic Theory, Volume 3 of SIAM Studies in Applied Math, SIAM, Philadelphia, 1981. 
[BMZ96] H.T. Banks, N.G. Medhin and Y. Zhang, A mathematical framework for curved active constrained layer structures: Wellposedness and approximatio, Num. Func. Analysis Optim., 17:1-22, 1996.

[BSW96] H.T. Banks, R.C. Smith and Y. Wang, Smart Material Structures: Modeling, Estimation and Control, Masson/J. Wiley, Paris/Chichester, 1996.

[Ch89] D.K. Cheng, Field and Wave Electromagnetics, Addison Wesley, Reading, MA, 1989 .

[CP78] R.F. Curtain and A.J. Pritchard, Infinite-Dimensional Linear Systems Theory, LN in Control and Info. Sci., Vol. 8, Springer Verlag, Berlin, 1978.

[CZ95] R.F. Curtain and H.J. Zwart, An Introduction to Infinite - Dimensional Linear Systems Theory, Springer Verlag, New York, 1995.

[El193] R.S. Elliott, Electromagnetics: History, Theory and Applications, IEEE Press, New York, 1993.

[FI90] R.H. Fabiano and K. Ito, Semigroup theory and numerical approximation for equations in linear viscoelasticity, SIAM J. Math. Anal., 21:374-393, 1990.

[Hop77] J. Hopkinson, The residual charge of the leyden jar, Phil. Trans. Roy. Soc. London, 167:599-626, 1877.

[Jac75] J.D. Jackson, Classical Electrodynamics, J. Wiley \& Sons, New York, 2nd edition, 1975.

[Paz83] A. Pazy, Semigroups of Linear Operators and Applications to Partial Differential Equations, Springer-Verlag, New York, 1983. 
[RMC93] J.R. Reitz, F.J. Melford and R.W. Christy, Foundations of Electromagnetic Theory, Addison Wesley, Reading, MA, 1993.

[vK93] B. van Keulen, $H_{\infty}$ Control for Distributed Parameter Systems: A State Space Approach, Birkhäuser, Boston, 1993.

[Wlo87] J. Wloka, Partial Differential Equations, Cambridge University Press, Cambridge, 1987. 